\title{
One-dimensional diffusion: Validity of various expressions for jump rates
}

\author{
S. J. Manzi, G. A. Ranzuglia, and V. D. Pereyra \\ Departamento de Física, Instituto de Física Aplicada (INFAP)-CONICET, Universidad Nacional de San Luis, Chacabuco \\ 917, 5700 San Luis, Argentina
}

(Received 27 July 2009; revised manuscript received 3 November 2009; published 17 December 2009)

\begin{abstract}
The coverage dependence of the one-dimensional collective diffusion coefficient is analyzed by using the gradient expansion of the local density. The transition probabilities are written as an expansion of the probabilities of the occupation configurations. Since the detail balance principle determines only a part of the diffusion terms in the expansion, different functional relations are proposed for these terms. The diffusion coefficient is obtained for various choices of these relations. However, some of them seem to be not physically sound and the diffusion coefficient does not behave properly. The range of validity of various expressions for the jump rates is determined and phase diagrams are shown. Besides that, it is shown that the transition state theory guarantees physically suitable behavior of the coefficient of one-dimensional diffusion.
\end{abstract}

DOI: 10.1103/PhysRevE.80.062104

PACS number(s): 05.50.+q, 68.43.De, 68.43.Jk

The diffusion of particles in low-dimensional systems is by now well understood (see, e.g., reviews [1] and original studies [2-8]). The potential energy for particle motion is determined in this case by a substrate and lateral particleparticle interactions. Often the particle-substrate interaction is sufficiently strong, particles are located primarily at the potential wells formed by the substrate and there are appreciable activation barriers for jumps of particles between the wells. In such cases, the diffusion can be described in the lattice-gas approximation. Beyond this approximation, the diffusion can be described, e.g., by using the FrenkelKontorova model [9].

In the framework of the lattice-gas approximation, the analysis of diffusion can be based on the linear-response theory [2] or gradient expansions of correlation functions $[10,11]$. The implementation of these two general approaches is however not straightforward and they do not provide compact expressions for the diffusion coefficient. A fairly general and relatively simple expression for the collective diffusion coefficient was phenomenologically proposed by Reed and Ehrlich (RE) [3]. In particular, they represented the diffusion coefficient as a product of thermodynamic and kinetic or dynamic factors. Physically, diffusion jumps represent the simplest Arrhenius processes. Such processes are customarily described by the transition state theory (TST) already many decades ago $[12,13]$. The advantage of TST is that it can be directly combined with, e.g., density-functional theory (DFT) in order to perform first-principles calculations or simulations. In the original TST, the effect of lateral particle-particle interactions on diffusion or other rate processes is not treated. Such interactions can however easily be introduced into the TST formalism. This strategy was realized for diffusion $[4,13]$ and other processes [13-15]. In particular, it was shown $[4,13]$ that following TST one can derive the $\mathrm{RE}$ expression for the diffusion coefficient.

More recently, many other approaches were used as well. In particular, a rather general approach not based on TST has recently been proposed by Payne and Kreuzer (PK) [8] for one-dimensional (1D) diffusion. They have exactly corroborated the RE factorization [3] for any range of interactions and for any form of the jump rates which satisfies detailed balance. As expected, this result agrees with that obtained in
Ref. [4]. The treatment has been extended to twodimensional (2D) systems, where PK showed that the RE factorization is not universal and is not applicable, e.g., for generalized hopping kinetics where initial- and final-state interactions are involved such as saddle-point interactions [16].

Although the approach proposed by PK is transparent and rather general, the physical meaning of the parameters they use is not straightforward. Moreover, for a given value of lateral interaction, there are two free parameters which allow different behavior of the 1D diffusion coefficient, including negative values. The goal of the present paper is to scrutinize the formal conditions used by PK and compare the results to TST for 1D diffusion. Concerning this subject, we may note the $1 \mathrm{D}$ case is of intrinsic interest. In addition, the general results for the $1 \mathrm{D}$ diffusion may be useful for applications, e.g., for describing adsorbate diffusion along steps [17] or on carbon nanotubes [18].

The RE factorization can be written as $[8,16]$,

$$
D(\theta, T)=\chi^{-1}\langle W\rangle
$$

where $\chi$ is the lattice-gas susceptibility and $\langle W\rangle=\frac{1}{2}\left\langle W_{i}^{>}\right.$ $\left.+W_{i+1}^{<}\right\rangle$is the average transition rate for 1D system, where

$$
\begin{aligned}
W_{i}^{>}= & J_{0} n_{i}\left(1-n_{i+1}\right)\left[\left(1-n_{i-1}\right)\left(1-n_{i+2}\right)\right. \\
& +\left(1+A_{1}\right) n_{i-1}\left(1-n_{i+2}\right)+\left(1+B_{1}\right)\left(1-n_{i-1}\right) n_{i+2} \\
& \left.+\left(1+A_{1}+B_{1}+C_{11}\right) n_{i-1} n_{i+2}\right] .
\end{aligned}
$$

Here one introduces the microscopic variable, which is $n_{i}$ $=1$ or 0 if the site is occupied or empty. In the last equation, the probability to jump to the right from site $i$ to $i+1$ is written in terms of the occupation configurations of all neighboring sites (a similar expression can be obtained for $\left.W_{i+1}^{<}\right)$. This form is illustrated in Fig. 1. The first term in Eq. (2) represents the jump of a particle which is isolated before and after the jump, with a rate $J_{0}$, the second term represents the separation of a particle from a neighbor with a rate $J_{0}\left(1+A_{1}\right)$ in the situations when the particle has no neighbors after the event, the third term represents the converse of the latter process, with a rate $J_{0}\left(1+B_{1}\right)$, and the last term represents the exchange of a particle between blocks. The elemen- 


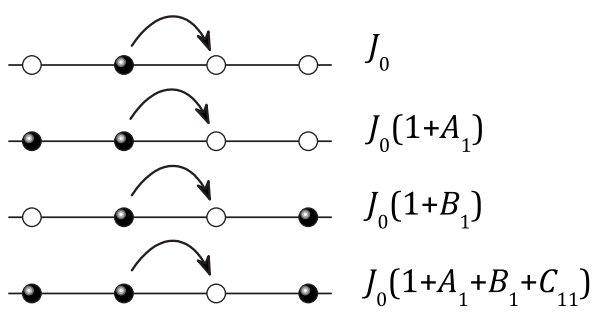

FIG. 1. The four relevant hopping processes and their rates for a one-dimensional lattice gas with nearest-neighbor interactions.

tary hopping rate, $J_{0}$, usually has the Arrhenius form.

Using the methodology developed by PK [8], one can finally write the diffusion coefficient in a diagrammatic form as

$$
D(\theta)=D_{0} \chi^{-1}\left[\langle\bullet \circ\rangle+A_{1}\langle\bullet \bullet\rangle+B_{1}\langle\bullet \circ \bullet\rangle+C_{11}\langle\bullet \bullet \bullet\rangle\right],
$$

where

$$
\begin{aligned}
& \left\langle\bullet_{i} \cdot{ }^{\bullet}{ }_{i+1} \cdots{ }^{\circ}{ }_{i+k}\right\rangle=\left\langle n_{i} n_{i+1} \cdots\left(1-n_{i+k}\right)\right\rangle \\
& =\sum_{\mathbf{n}} n_{i} n_{i+1} \cdots\left(1-n_{i+k}\right) P(\{\mathbf{n}\}, \mathbf{t}) .
\end{aligned}
$$

Here, $P(\{\mathbf{n}\}, t)$ represents the probability that a given microscopic configuration $\{\mathbf{n}\}=\left(n_{1}, n_{2}, \ldots, n_{N}\right)$ is realized at time $t$, where $N$ is the total number of sites in the line. The inverse of the susceptibility is

$$
\chi^{-1}=\frac{\partial \beta \mu}{\partial \theta}=[\alpha \theta(1-\theta)]^{-1},
$$

where $\theta$ is the coverage and $\alpha$ is related to the chemical potential by

$$
\exp [\beta \mu]=\frac{\alpha-1+2 \theta}{\alpha+1-2 \theta} \exp [\beta V],
$$

where $\beta=1 / k_{B} T$ and $V$ is the nearest-neighbor lateral interaction.

The detailed balance principle puts one condition on the three coefficients, $A_{1}, B_{1}$, and $C_{11}$, namely,

$$
\left(1+A_{1}\right)=\left(1+B_{1}\right) e^{\beta V} .
$$

Different scenarios were analyzed by PK [8] for various choices of diffusion kinetics. The first one is the so-called initial-state interaction. This kinetic scheme is characterized by $B_{1}=C_{11}=0$ (case I). The second kinetic scheme is the final-state interaction, where $A_{1}=C_{11}=0$ (case II). The diffusion coefficient for these two cases is positive for any value of the lateral interaction.

Then, PK analyzed the case of saddle-point interactions. To define this scheme, they proposed a linear relation between the coefficients as

$$
A_{1}=-\gamma B_{1},
$$

where $\gamma$ is a free parameter ( $\mathrm{PK}$ consider that this parameter is of order unity, i.e., $B 1 \simeq-A_{1}$ ). Taking into account the detailed balance principle, they obtained

$$
B_{1}=\frac{1-\exp (\beta V)}{\gamma+\exp (\beta V)}
$$

Furthermore, no restriction is imposed on $C_{11}$. Therefore, they analyzed three different cases, i.e., $C_{11}=0$ (case III), $C_{11}=-A_{1}$ (case IV), and $C_{11}=-B_{1}$ (case V). As observed in Ref. [8], the normalized diffusion coefficient as a function of the coverage is always bigger than unity $[D(\theta) / D(0)>1]$ for repulsive lateral interactions and smaller than unity $[D(\theta) / D(0)<1]$ for attractive lateral interactions, with the exception of case II, where the behavior is the opposite.

However, in the framework of the PK formulation [Eq (3)], there is no restriction on $\gamma$, certainly it can take any value. The behavior of the diffusion coefficient is reasonable for any positive value of $\gamma$. For negative values of $\gamma$, there are however combinations of parameters $\gamma$ and $V$ that lead to the negative diffusion coefficient.

It is well known that no phase transitions occur in 1D systems, therefore the diffusion coefficient must be positive. The detailed balance principle is not enough to guarantee that the diffusion coefficient is positive. Then, to satisfy this physical condition, one should consider that the jump rate constants in Eq. (2) should be positive or null, so the following inequalities must be fulfilled:

$$
\begin{aligned}
& \left(1+A_{1}\right) \geq 0, \\
& \left(1+B_{1}\right) \geq 0,
\end{aligned}
$$

and

$$
\left(1+A_{1}+B_{1}+C_{11}\right) \geq 0 .
$$

These relations are similar that those obtained for the adsorption and desorption kinetics in Ref. [15]. Taking into account
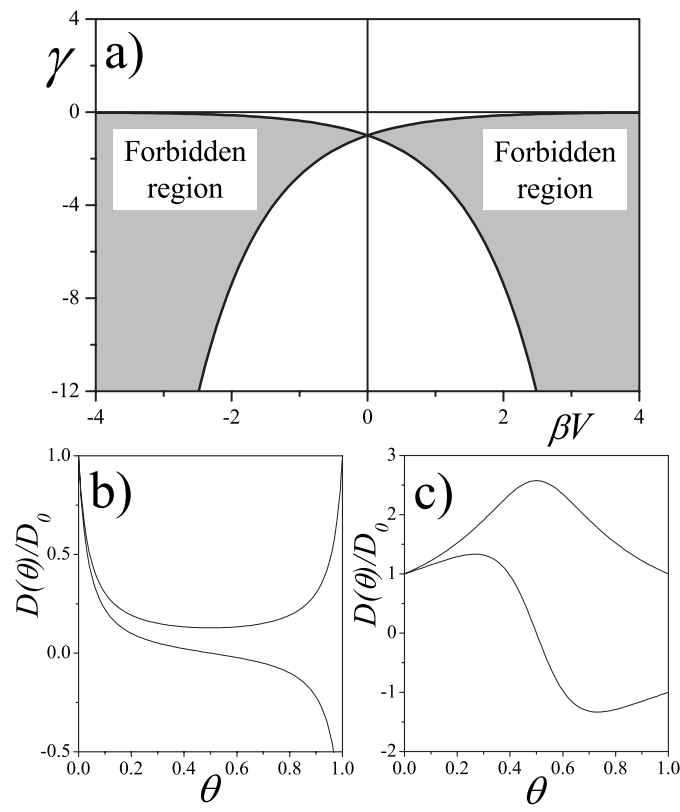

FIG. 2. (a) Diagram $\gamma$ vs $\beta V$ corresponding to case III. The coverage dependence of the diffusivity, $D$, for varying firstneighbor interaction strength and $\gamma$ parameter. (b) $\beta V=-2$ and $\gamma$ $=1,-1$. (c) $\beta V=2$ and $\gamma=1,-1$. 

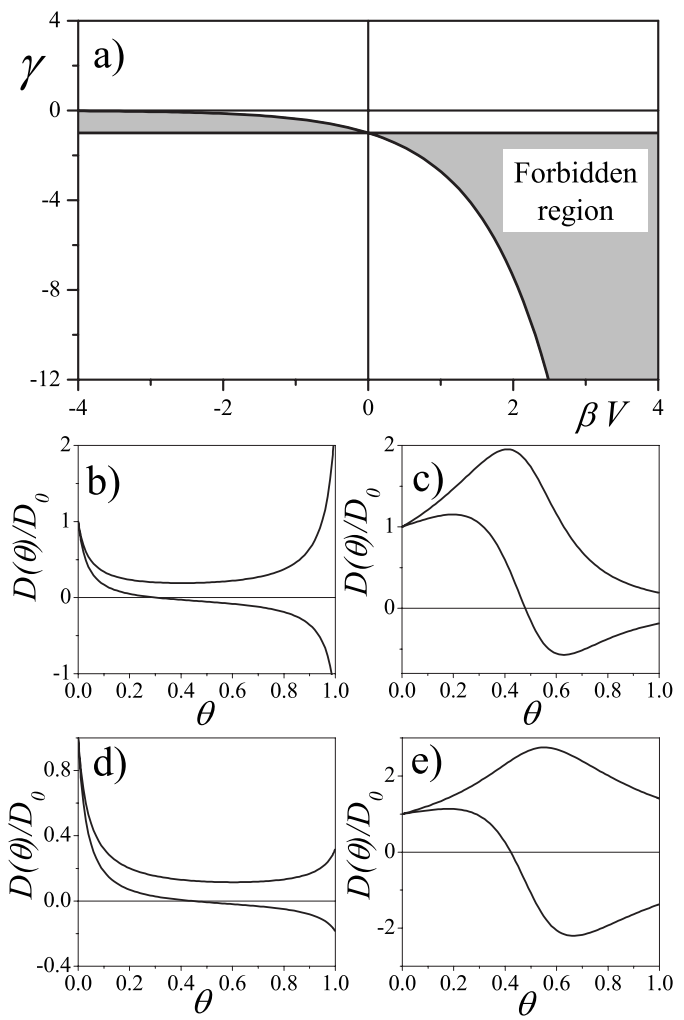

FIG. 3. (a) Diagram $\gamma$ vs $\beta V$ corresponding to cases IV and V. The coverage dependence of the diffusivity, $D$, for varying firstneighbor interaction strength and $\gamma$ parameter. (b) Case IV: $\beta V=$ -2 and $\gamma=0.5,-0.5$. (c) Case IV: $\beta V=2$ and $\gamma=0.5,-2$. (d) Case V: $\beta V=-2$ and $\gamma=0.5,-0.5$. (e) Case IV: $\beta V=2$ and $\gamma=0.5,-2$.

these constraints, it is possible to build a phase diagram showing the values of $\gamma$ and $V$ where the diffusion coefficient is positive or negative. In Fig. 2(a), the phase diagram $(\gamma$ vs $V)$ corresponding to case III is plotted. In Figs. 2(b) and 2(c), the diffusion coefficients as a function of coverage are plotted for two different pairs of values of $\gamma$ and $V$. As is observed, the diffusion coefficient takes negative values for certain values of coverage. In Fig. 3, the phase diagram corresponding to cases IV and V is plotted. Similar behavior is observed for the diffusion coefficient corresponding to the forbidden regions.

On the other hand, TST $[4,13]$ provides a way to obtain the rate constants of the diffusion processes considering the saddle-point interaction. Specifically, in the framework of the TST, the average transition rate given in Eq. (2) can be written as

$$
\begin{aligned}
W_{i}^{>}= & J_{0}\left\{P_{0,0}^{\langle\bullet \bullet}+P_{1,0}^{\langle\bullet\rangle} \exp \left[\beta\left(\epsilon_{1}-\epsilon_{1}^{*}\right)\right]+P_{0,1}^{\langle\bullet \bullet\rangle} \exp \left(-\beta \epsilon_{1}^{*}\right)\right. \\
& \left.+P_{1,1}^{(\bullet \bullet)} \exp \left[\beta\left(\epsilon_{1}-2 \epsilon_{1}^{*}\right)\right]\right\}
\end{aligned}
$$

where $P_{i, j}^{\langle\bullet \bullet\rangle}$ is the probability to have an occupied site with the right neighbor site empty and the neighborhood denoted by the indexes $i, j ; \epsilon_{1}\left(\epsilon_{1}^{*}\right)$ is the lateral interaction energy for the diffusion jumps in the ground (activated or transition) state.

In order to obtain Eq. (3) by using TST formulation, the coefficients must fulfill the following relations:
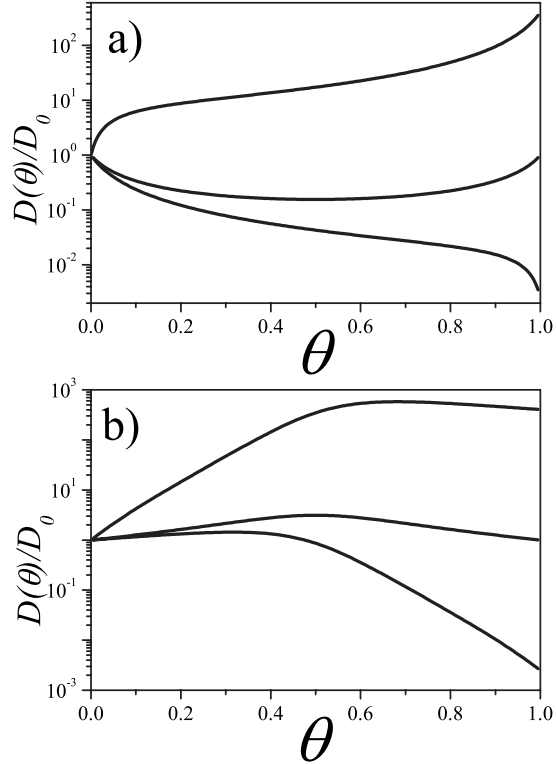

FIG. 4. The coverage dependence of the diffusivity, $D$, for varying $\delta$ parameter using TST. (a) $\beta V=-2$ : top to bottom $\delta=2,0.5$, -1 . (b) $\beta V=2$ : top to bottom $\delta=-1,0.5,2$.

$$
\begin{gathered}
A_{1}=\exp \left[\beta\left(\epsilon_{1}-\epsilon_{1}^{*}\right)\right]-1, \\
B_{1}=\exp \left(-\beta \epsilon_{1}^{*}\right)-1,
\end{gathered}
$$

and

$$
C_{11}=\exp \left[\beta\left(\epsilon_{1}-2 \epsilon_{1}^{*}\right)\right]-1-A_{1}-B_{1} .
$$

By definition, we have $\epsilon_{1}=V$. The interaction in the activated state can be represented as $\epsilon_{1}^{*}=\delta V$, where $\delta$ is the proportionality factor (like in the Brönsted-Polanyi correlations). Then, the three coefficients involved into the PK formalism can be written as

$$
\begin{gathered}
A_{1}=e^{(1-\delta) \beta V}-1, \\
B_{1}=e^{-\delta \beta V}-1,
\end{gathered}
$$

and

$$
C_{11}=e^{(1-2 \delta) \beta V}-e^{(1-\delta) \beta V}-e^{-\delta \beta V}+1 .
$$

Note that in the present TST formulation, there is only one free parameter, $\delta$. On the other hand, the diffusion coefficient is always positive for any value of lateral interaction, as expected. Moreover, the initial-state interactions (case I) are obtained making $\delta=0$. Similarly, the final-state interactions (case II) are recovered making $\delta=1$. The particle-hole symmetry can be obtained making $\delta=1 / 2$.

On the other hand, neither the TST formulation nor the detailed balance principle imposes any restriction on $\delta$. However, when this parameter takes a value out of the interval $[0,1]$, the behavior of the diffusion coefficient as a function of the coverage is somewhat unusual from our point of view. In Figs. 4(a) and 4(b), we have plotted $D(\theta) / D(0)$ vs $\theta$ for attractive $\beta V=-2$ and repulsive $\beta V=2$ lateral interactions, respectively. In these figures, different values of parameter $\delta$ are used. As one can observe, for attractive lateral interaction 
and $\delta=2, D(\theta) / D(0)$ increases with increasing coverage (this behavior is proper of repulsive interaction) and for repulsive lateral interaction and $\delta=2$ decreases with coverage $D(\theta) / D(0)<1$, which is characteristic of attractive lateral interactions.

Summarizing, in this report, the coverage dependence of the one-dimension collective diffusion coefficient is analyzed in the framework of the model introduced by PK. It is observed that this formulation is rather general containing almost all the possible scenarios for the jump kinetic processes. However, for certain value of the coefficients, the diffusion coefficient does not behave properly taking negative values. Specifically, the detailed balance principle is not sufficient to ensure that the diffusion coefficient is positive and accordingly, further restrictions are needed on the parameters to satisfy this condition. As a result, it is possible to build a phase diagram showing the values of $\gamma$ and $V$ where the diffusion coefficient is positive or negative.

Alternatively, the TST is used to calculate the diffusion coefficient. It is shown that in the framework of this theory, only one free parameter is necessary to calculate the diffusion coefficient (instead of two in the former model). Although this theory guarantees that the diffusion coefficient is always positive for any value of the lateral interaction, neither the TST nor the detailed balance principle imposes restriction on the free parameter. Therefore, for $\delta<0$ or $\delta>1$, the behavior of the diffusion coefficient can be somewhat unusual.

This work is partially supported by the CONICET (Argentina).
[1] J. W. Haus and K. Kehr, Phys. Rep. 150, 263 (1987); R. Gomer, Rep. Prog. Phys. 53, 917 (1990); T. Ala-Nissila, R. Ferrando, and S. C. Ying, Adv. Phys. 51, 949 (2002); G. Zgrablich, in Equilibria and Dynamics of Gas Adsorption on Heterogeneous Surfaces, edited by W. Rudzinski, W. A. Steele, and G. Zgrablich (Elsevier, Amsterdam, 1997); J. V. Barth, Surf. Sci. Rep. 40, 75 (2000); F. Nieto et al., Defect Diffus. Forum 162, 59 (1998); A. G. Naumovets and Z. Y. Zhang, Surf. Sci. 500, 414 (2002); G. Antczak and G. Ehrlich, ibid. 589, 52 (2005).

[2] W. Zwerger, Z. Phys. B: Condens. Matter 42, 333 (1981).

[3] D. A. Reed and G. Ehrlich, Surf. Sci. 102, 588 (1981); 105, 603 (1981).

[4] V. P. Zhdanov, Surf. Sci. 149, L13 (1985).

[5] A. V. Myshlyavtsev, A. A. Stepanov, C. Uebing, and V. P. Zhdanov, Phys. Rev. B 52, 5977 (1995); C. Uebing and V. P. Zhdanov, Phys. Rev. Lett. 80, 5455 (1998); J. Chem. Phys. 109, 3197 (1998); F. M. Bulnes, V. D. Pereyra, and J. L. Riccardo, Phys. Rev. E 58, 86 (1998); A. J. Ramirez-Pastor, T. P. Eggarter, V. D. Pereyra, and J. L. Riccardo, Phys. Rev. B 59, 11027 (1999).

[6] P. Argyrakis, A. A. Chumak, and M. Maragakis, Phys. Rev. B 71, 224304 (2005); 76, 054209 (2007); P. Argyrakis, M. Maragakis, O. Chumak, and A. Zhugayevych, ibid. 74, 035418 (2006); P. Argyrakis and A. A. Chumak, ibid. 66, 054303 (2002).

[7] L. Badowski, M. A. Zaluska-Kotur, and Z. W. Gortel, Phys. Rev. B 72, 245413 (2005); M. A. Zaluska-Kotur and Z. W. Gortel, ibid. 72, 235425 (2005); 74, 045405 (2006).

[8] S. H. Payne and H. J. Kreuzer, Phys. Rev. B 75, 115403
(2007).

[9] O. M. Braun, T. Dauxois, M. V. Paliy, and M. Peyrard, Phys. Rev. E 55, 3598 (1997).

[10] K. Kawasaki, Phys. Rev. 145, 224 (1966).

[11] Z. Chvoj, J. Phys.: Condens. Matter 12, 2135 (2000).

[12] S. Glasstone, K. J. Laidler, and H. Eyring, The Theory of Rate Processes (McGraw-Hill, New York, 1941).

[13] V. P. Zhdanov, Elementary Physicochemical Processes on Solid Surfaces (Plenum, New York, 1991).

[14] V. P. Zhdanov, Surf. Sci. 111, 63 (1981); 111, L662 (1981); 123, 106 (1982); 133, 469 (1983); 157, L384 (1985); 165, L31 (1986); 171, L461 (1986); 209, 523 (1989); Surf. Sci. Rep. 12, 185 (1991); B. Li, C.-S. Zhang, V. P. Zhdanov, and P. R. Norton, Surf. Sci. 322, 373 (1995); V. P. Zhdanov and B. Kasemo, ibid. 412-413, 527 (1998).

[15] S. J. Manzi, R. E. Belardinelli, G. Costanza, and V. D. Pereyra, Phys. Rev. E 79, 021103 (2009); S. J. Manzi, V. J. Huespe, R. E. Belardinelli, G. Costanza, and V. D. Pereyra, ibid. 80, 051112 (2009).

[16] S. H. Payne and H. J. Kreuzer, Phys. Rev. B 77, 121403(R) (2008); J. Phys.: Condens. Matter 21, 134013 (2009).

[17] R. Butz and H. Wargner, Surf. Sci. 87, 85 (1979); M. V. Arena et al., ibid. 325, 151 (1995); V. P. Zhdanov, Phys. Lett. A 137, 409 (1989); J. Merikoski and S. C. Ying, Phys. Rev. B 56, 2166 (1997); 58, 15912 (1998); M. Mašin et al., Surf. Sci. 529, L256 (2003); 544, L703 (2003); 566-568, 143 (2004); Z. Chvoj et al., J. Stat. Mech.: Theory Exp. (2006) P10003.

[18] A. Kirakosian, R. Bennewitz, F. J. Himpsel, and L. W. Bruch, Phys. Rev. B 67, 205412 (2003); Y. Gan et al., New. J. Phys. 10, 023022 (2008). 